\title{
ON SPECTRA OF LÜDERS OPERATIONS
}

\author{
GABRIEL NAGY
}

\begin{abstract}
We show that the all eigenvalues of certain generalized Lüders operations are non-negative real numbers, in two cases of interest. In particular, given a commuting $n$-tuple $\mathcal{A}=\left(A_{1}, \ldots, A_{n}\right)$ consisting of positive operators on a Hilbert space $\mathcal{H}$, satisfying $\sum_{j=1}^{n} A_{j}=I$, we show that the spectrum of the Lüders operation:

$$
\Lambda_{\mathcal{A}}: \mathcal{B}(\mathcal{H}) \ni X \longmapsto \sum_{j=1}^{n} A_{j}^{1 / 2} X A_{j}^{1 / 2} \in \mathcal{B}(\mathcal{H}),
$$

is contained in $[0, \infty)$, so the only solution of the equation $\Lambda_{\mathcal{A}}(X)=I-X$ is the "expected" one $X=\frac{1}{2} I$.
\end{abstract}

\section{INTRODUCTION}

An important question in Quantum Mechanics is concerned with the effects of a (quantum) measurement on the entity that is being measured. In connection with this problem, the Lüders operations play a significant role. The traditional framework for describing this problem is operator-theoretic. For a Hilbert space $\mathcal{H}$, one defines the space of effects as $\mathcal{E}(\mathcal{H})=\{A \in \mathcal{B}(\mathcal{H}): 0 \leq A \leq I\}$, and one defines a discrete finite quantum measurement as an $n$-tuple $\mathcal{A}=\left(A_{1}, \ldots, A_{n}\right) \in \mathcal{E}(\mathcal{H})^{n}$, satisfying the probability density condition $\sum_{k=1}^{n} A_{k}=I$. (One understands $\mathcal{A}$ as a "device" for measuring the occurrence of one out of $n$ possible outcomes, with each $A_{k}$ ' as a "filter" aimed at deciding if $k^{\text {th }}$ outcome occurs.) The set of physical states is understood as the space $\mathcal{S}(\mathcal{H})=\{S \in \mathcal{B}(\mathcal{H}): S \geq 0$, Trace $(S)=1\}$. (In the language of operator algebras, $\mathcal{S}(\mathcal{H})$ is the space of normal states on the von Neumann algebra $\mathcal{B}(\mathcal{H})$.) Given a quantum measurement $\mathcal{A}=\left(A_{1}, \ldots, A_{n}\right)$ and some index $k \in\{1, \ldots, n\}$, the probability that $k^{\text {th }}$ outcome occurs in some state $S$ is: $P_{S}\left(A_{k}\right)=\operatorname{Trace}\left(S A_{k}\right)$. (By the probability density condition the sum of these 
probabilities is indeed equal to 1.) The new physical state after the measurement (defined by $\mathcal{A}$ ) is performed, is defined using the Lüders operation.

$$
\Lambda_{\mathcal{A}}(S)=\sum_{k=1}^{n} A_{k}^{1 / 2} S A_{k}^{1 / 2}
$$

The Lüders operation is regarded initially as a an affine self-map of the convex set $\mathcal{S}(\mathcal{H})$. It can be regarded also as a linear continuous map $\Lambda_{\mathcal{A}}: \mathcal{C}_{1}(\mathcal{H}) \rightarrow \mathcal{C}_{1}(\mathcal{H})$, defined again by $(1)$, where $\mathcal{C}_{1}(\mathcal{H})$ denotes the space of trace-class operators. (It is well-known that $\mathcal{C}_{1}(\mathcal{H})$ is the linear span of $\mathcal{S}(\mathcal{H})$.) Using this language, the question whether a state $S$ is undisturbed by the measurement $\mathcal{A}$ becomes equivalent to the fact that $S$ is a solution of the equation

$$
\Lambda_{\mathcal{A}}(S)=S
$$

By the generalized Lüders Theorem proven in [2] we know that a state $S \in \mathcal{S}(\mathcal{H})$ is a solution of (2), if and only $S$ is compatible with $\mathcal{A}$, i.e. $S$ commutes with all $A_{k}$ 's. The more interesting problem is the dual one, when the Lüders operation is in fact extended to a map $\Lambda_{\mathcal{A}}: \mathcal{B}(\mathcal{H}) \rightarrow \mathcal{B}(\mathcal{H})$, defined again by $(1)$. It is not hard to see that $\Lambda_{\mathcal{A}}$ is also an affine self-map of $\mathcal{E}(\mathcal{H})$, so now we can focus on the equation

$$
\Lambda_{\mathcal{A}}(B)=B, \quad B \in \mathcal{E}(\mathcal{H})
$$

In this dual approach it has been beneficial (for physical interpretations) to examine the sequential product of effects, defined in [5] as

$$
A \circ B=A^{1 / 2} B A^{1 / 2}, \quad A, B \in \mathcal{E}(\mathcal{H})
$$

which clearly takes values in $\mathcal{E}(\mathcal{H})$. With this terminology, the Lüders operation is simply $\Lambda_{\mathcal{A}}(B)=\sum_{k=1}^{n} A_{k} \circ B$. If we think of two effects $A$ and $B$ as "filters," each perhaps parts of two quantum measurements $\mathcal{A}$ and $\mathcal{B}$ respectively, then $A \circ B$ is part of the so-called sequential product measurement $\mathcal{A} \circ \mathcal{B}$ (which Gudder defined in [3]), namely the " $A$ followed by $B$ filter." In his programme for studying the 
sequential product of measurements, Gudder was lead to the investigation of the equation

$$
\Lambda_{\mathcal{A}}(B)=I-B, \quad B \in \mathcal{E}(\mathcal{H}) .
$$

Both equations (3) and (4) are naturally interpreted as extremal cases of the disturbance problem: whether the measurement $\mathcal{A}$ does not disturb $B$, or disturbs it completely into its supplement $I-B$.

The equation (3) was extensively investigated by Arias, Gheondea and Gudder in [1], motivated by an older result of Gudder and this author (see [5]), where it was shown that, in the case $n=2$, that is, when one uses a yes-no measurement $\mathcal{A}=(A, I-A), A \in \mathcal{E}(\mathcal{H})$, then solutions of (3) are precisely the effects $B \in \mathcal{E}(\mathcal{H})$ which commute with $A$. A major unresolved problem concerning (3) is to give a sufficient condition on an arbitrary measurement $\mathcal{A}$ (with $n>2$ elements), for which the solution set of $(3)$ is $\mathcal{E}(\mathcal{H}) \cap \mathcal{A}^{\prime}$, where $\mathcal{A}^{\prime}=\left\{B \in \mathcal{B}(\mathcal{H}): A_{k} B=B A_{k}, \forall k\right\}$ is the commutant of $\mathcal{A}$. In [1] it has been shown that a necessary condition is injectivity of $\mathcal{A}^{\prime}$, so a "bold conjecture" would be that this is also sufficient. The test case for this conjecture is when $\mathcal{A}$ is abelian, and even in this case there is no proof available.

In connection with (4) it is not surprising that in the case $n=2$, when the measurement is again of the form $\mathcal{A}=(A, I-A), A \in \mathcal{E}(\mathcal{H})$, the solution set is a singleton: $\left\{\frac{1}{2} I\right\}$. Gudder [3] proved this in the finite dimensional case, and Wang, $\mathrm{Du}$ and Zuo [8] generalized it to the infinite dimensional case. It is worth pointing out that in both proofs, the fact that $\mathcal{A}$ is abelian (forced by the fact that $\mathcal{A}$ has two elements $A$ and $I-A$ ) plays an important role. One may conjecture that the same conclusion holds when $\mathcal{A}$ is abelian, and we will confirm in this article that this statement is correct. As it turns out, if the abelian version of the "bold conjecture" holds (i.e. if $\mathcal{A}$ is abelian, then the solution set of $(3)$ is $\mathcal{E}(\mathcal{H}) \cap \mathcal{A}^{\prime}$ ), then indeed the equation (4) has only one solution (the "expected" one), in the abelian case. To see this, all one needs to do is to notice the fact that (4) implies that $B$ is a 
solution of the equation

$$
\Lambda_{\mathcal{A} \circ \mathcal{A}}(B)=B
$$

and $\mathcal{A} \circ \mathcal{A}=\left(A_{j} A_{k}\right)_{j, k=1}^{n}$ is abelian.

The purpose of this note is to investigate the spectrum of $\Lambda_{\mathcal{A}}$. Since the probability density condition on $\mathcal{A}$ implies that $\Lambda_{\mathcal{A}}$ is unital, i.e. $\Lambda_{\mathcal{A}}(I)=I$, it is pretty obvious that, if we substitute $X=B-\frac{1}{2} I$ in (3), then we obtain

$$
\Lambda_{\mathcal{A}}(X)=-X,
$$

so if $B \neq \frac{1}{2} I$, then -1 would be an eigenvalue for $\Lambda_{\mathcal{A}}$. In the cases of interest suggested in [1], we are going to show that not only -1 fails to be an eigenvalue, but in fact all eigenvalues of $\Lambda_{\mathcal{A}}$ are non-negative real numbers, thus offering two generalizations of the results of Gudder, Wang, Du and Zuo. Our approach will employ two results (Theorems 1 and 2 below) concerning the spectrum of certain generalized Lüders operations, namely. of the form

$$
\Theta(X)=\sum_{k=1}^{n} A_{k} X B_{k}
$$

involving certain operators $A_{1}, \ldots, A_{n}, B_{1}, \ldots, B_{n}$ with non-negative spectrum. In the abelian case (when all $A$ 's and all $B$ 's commute with each other) one does not even require them to be self-adjoint.

\section{RESUlts}

The first result provides the needed spectral estimate in the abelian case.

Theorem 1. Let $\mathfrak{A}$ be a unital Banach algebra, and let $\left(x_{1}, \ldots, x_{n}, y_{1}, \ldots, y_{n}\right)$ be a 2 -tuple of commuting elements in $\mathfrak{A}$. If the spectra of all $x$ 's and all $y$ 's are contained in $[0, \infty)$, then the linear operator $\Theta: \mathfrak{A} \rightarrow \mathfrak{A}$, defined by

$$
\Theta(a)=\sum_{k=1}^{n} x_{k} a y_{k}, \quad a \in \mathfrak{A},
$$

is continuous and has its spectrum contained in $[0, \infty)$. 
Proof. Continuity is pretty obvious. Define, for any $b \in \mathfrak{A}$, the linear operators $L_{b}, R_{b}: \mathfrak{A} \rightarrow \mathfrak{A}$ by $L_{b} a=b a$ and $R_{b} a=a b, \forall a \in \mathfrak{A}$. It is well known that, for any $b \in \mathfrak{A}$, the linear operators $L_{b}$ and $R_{b}$ are both continuous (in fact one has $\left.\left\|L_{b}\right\|=\left\|R_{b}\right\|=\|b\|\right)$, and, as elements in the Banach algebra $\mathcal{L}(\mathfrak{A})$ of all linear continuous operators on $\mathfrak{A}$, their spectra are given by

$$
\operatorname{Spec}_{\mathcal{L}(\mathfrak{A})}\left(L_{b}\right)=\operatorname{Spec}_{\mathcal{L}(\mathfrak{A})}\left(R_{b}\right)=\operatorname{Spec}_{\mathfrak{A}}(b) .
$$

In particular, all spectra $\operatorname{Spec}_{\mathcal{L}(\mathfrak{A})}\left(L_{x_{j}}\right), \operatorname{Spec}_{\mathcal{L}(\mathfrak{A})}\left(R_{y_{j}}\right), j=1, \ldots, n$ are contained in $[0, \infty)$.

Consider then sub-algebra $\mathfrak{M}$ generated by the set

$$
\mathcal{S}=\left\{I, L_{x_{1}}, \ldots, L_{x_{n}}, R_{y_{1}}, \ldots, R_{y_{n}}\right\}
$$

which is a unital abelian sub-algebra of $\mathcal{L}(\mathfrak{A})$. The fact that $\mathfrak{M}$ is abelian follows easily from two simple observations:

(i) $R_{b} L_{c}=L_{c} R_{b}, \forall b, c \in \mathfrak{A}$;

(ii) if $b, c \in \mathfrak{A}$ commute, then $R_{b}$ and $R_{c}$ commute, and likewise, $L_{b}$ and $L_{c}$ commute.

If we define now the enhanced set

$$
\mathcal{E}=\mathfrak{M} \cup \bigcup_{T \in \mathfrak{M}}\left\{(T-\lambda I)^{-1}: \lambda \in \mathbb{C} \backslash \operatorname{Spec}_{\mathcal{L}(\mathfrak{A})}(T)\right\},
$$

then $\mathcal{E}$ is again abelian, and so is the Banach sub-algebra $\mathfrak{B} \subset \mathcal{L}(\mathfrak{A})$, generated by $\mathcal{E}$. By construction, we have the spectral permanence equalities

$$
\operatorname{Spec}_{\mathfrak{B}}(T)=\operatorname{Spec}_{\mathcal{L}(\mathfrak{A})}(T), \quad \forall T \in \mathfrak{M}
$$

Consider now the Gelfand transform $\mathfrak{B} \ni T \longmapsto \hat{T} \in C\left(\Gamma_{\mathfrak{B}}\right)$, defined by $\hat{T}(\gamma)=$ $\gamma(T), \forall T \in \mathfrak{B}, \gamma \in \Gamma_{\mathfrak{B}}$, where $\Gamma_{\mathfrak{B}}$ denotes character space. It is well-known that, for every $T \in \mathfrak{B}$ one has the equality

$$
\operatorname{Spec}_{\mathfrak{B}}(T)=\text { Range } \hat{T} \text {. }
$$


In particular, if consider the elements $X_{j}=L_{x_{j}}, Y_{j}=R_{y_{j}} \in \mathfrak{M}, j=1, \ldots, n$, so that we have $\Theta=\sum_{k=1}^{n} X_{j} Y_{j} \in \mathfrak{M}$, then using (5) we obtain

$$
\operatorname{Spec}_{\mathcal{L}(\mathfrak{A})}(\Theta)=\operatorname{Spec}_{\mathfrak{B}}(\Theta)=\operatorname{Range}\left(\sum_{j=1}^{n} \hat{X}_{j} \hat{Y}_{j}\right)
$$

Using again (5), we know that $\operatorname{Spec}_{\mathfrak{B}}\left(X_{j}\right), \operatorname{Spec}_{\mathfrak{B}}\left(Y_{j}\right) \subset[0, \infty), j=1, \ldots, n$, which by (6) means that the continuous functions $\hat{X}_{1}, \ldots, \hat{X}_{n}, \hat{Y}_{1}, \ldots, \hat{Y}_{n} \in C\left(\Gamma_{\mathfrak{B}}\right)$ all have their ranges in $[0, \infty)$. It now follows immediately that the function $\sum_{j=1}^{n} \hat{X}_{j} \hat{Y}_{j} \in$ $C\left(\Gamma_{\mathfrak{B}}\right)$ also has its range in $[0, \infty)$, so going back to $(7)$, we get the desired inclusion $\operatorname{Spec}_{\mathcal{L}(\mathfrak{A})}(\Theta) \subset[0, \infty)$.

Corollary 1. Assume $\mathfrak{A}$ is a unital Banach algebra, and $x_{1}, \ldots, x_{n}, y_{1}, \ldots, y_{n} \in \mathfrak{A}$ are commuting elements, each having its spectrum contained in $[0, \infty)$, such that $\sum_{j=1}^{n} x_{j} y_{j}=1$. For any $\lambda \in \mathbb{C}$ and any $\mu \in \mathbb{C} \backslash[0, \infty)$, the equation

$$
\sum_{j=1}^{n} x_{j} z y_{j}=\mu z+\lambda(1-\mu) 1, \quad z \in \mathfrak{A}
$$

has only one solution: $z=\lambda 1$.

Proof. By Theorem 1, the linear continuous map

$$
\Theta: \mathfrak{A} \ni a \longmapsto \sum_{j=1}^{n} x_{j} a y_{j} \in \mathfrak{A} .
$$

has its spectrum contained in $[0, \infty)$. In particular, for any $\mu \in \mathbb{C} \backslash[0, \infty)$ the linear continuous map $\Theta-\mu \mathrm{Id}: \mathfrak{A} \rightarrow \mathfrak{A}$ is invertible, thus the only solution of the equation

$$
\Theta(a)=\mu a
$$

is the trivial one: $a=0$.

Suppose now $z$ is a solution of (8). Since $\Theta(1)=1$, if we subtract $\Theta(\lambda 1)=\lambda 1$ from both sides of (8), we get

$$
\Theta(z-\lambda 1)=\mu(z-\lambda 1)
$$

which means that $a=z-\lambda 1$ is a solution of (9), so we indeed have $z=\lambda 1$. 
Corollary 2. If $\mathcal{H}$ is a Hilbert space, and $\mathcal{A}=\left(A_{1}, \ldots, A_{n}\right) \in \mathcal{E}(\mathcal{H})^{n}$ is an abelian discrete finite quantum measurement, then the equation

$$
\Lambda_{\mathcal{A}}(B)=I-B, \quad B \in \mathcal{B}(\mathcal{H})
$$

has exactly one solution: $B=\frac{1}{2} I$.

Proof. Immediate from Corollary 1 , applied to $\mathfrak{A}=\mathcal{B}(\mathcal{H})$ with $x_{j}=y_{j}=A_{j}^{1 / 2}$, $\lambda=\frac{1}{2}$ and $\mu=-1$.

We now turn our attention to the second special case, for which we introduce the following terminology.

Definition. Given a von Neumann algebra $\mathfrak{M}$, we call a von Neumann sub-algebra $\mathfrak{A} \subset \mathfrak{M}$ faithfully injected in $\mathfrak{M}$, if

(i) $\mathfrak{A}$ contains the unit of $\mathfrak{M}$, and

(ii) there exists a faithful conditional expectation $E$ of $\mathfrak{M}$ onto $\mathfrak{A}$.

Recall that, given unital $\mathrm{C}^{*}$-algebras $\mathfrak{A} \subset \mathfrak{M}$ with common unit, the condition that a linear map $E: \mathfrak{M} \rightarrow \mathfrak{M}$ is a conditional expectation of $\mathfrak{M}$ onto $\mathfrak{A}$ means that $E$ is a contractive idempotent (i.e. $\|E\|=1$ and $E \circ E=E$ ) with Range $E=\mathfrak{A}$. According to Tomyiama's Theorem ([7]), such a map $E$ is completely positive and also satisfies the condition

$$
E(a y b)=a E(x) b, \quad \forall a, b \in \mathfrak{A}, x \in \mathfrak{M} .
$$

The condition that $E$ is faithful means that, whenever $x \in \mathfrak{M}$ is a positive element such that $E(x)=0$, it follows that $x=0$.

With this terminology, one has the following result.

Theorem 2. Assume $\mathfrak{M}$ is a von Neumann algebra, and $\mathfrak{A}$ is a finite faithfully injected von Neumann sub-algebra of $\mathfrak{M}$. If $a_{1}, \ldots, a_{n}, b_{1}, \ldots, b_{n} \in \mathfrak{A}$ are positive elements, then all the eigenvalues of the linear operator

$$
\Theta: \mathfrak{M} \ni x \longmapsto \sum_{j=1}^{n} a_{j} x b_{j} \in \mathfrak{M}
$$


are non-negative real numbers.

Proof. Fix a faithful conditional expectation $E$ of $\mathfrak{M}$ onto $\mathfrak{A}$. Let $\mathfrak{Z}$ denote the center of the (finite) von Neumann algebra $\mathfrak{A}$, and let

$$
\mathfrak{A} \ni a \longmapsto a^{\natural} \in \mathfrak{Z}
$$

denote the center valued trace. It is well known (see for instance [6] with references therein) that (10) is a faithful conditional expectation of $\mathfrak{A}$ onto $\mathfrak{Z}$, so the map

$$
\mathfrak{M} \ni x \longmapsto E(x)^{\natural} \in \mathfrak{Z}
$$

is now a faithful conditional expectation of $\mathfrak{M}$ onto $\mathfrak{Z}$. Identify, using the Gelfand transform, $\mathfrak{Z} \simeq C\left(\Gamma_{\mathfrak{Z}}\right)$, and define, for every $\gamma \in \Gamma_{\mathfrak{Z}}$ the state $\phi_{\gamma}: \mathfrak{M} \rightarrow \mathbb{C}$ by

$$
\phi_{\gamma}(x)=\gamma\left(E(x)^{\natural}\right) .
$$

Denote, for every $\gamma \in \Gamma_{\mathfrak{Z}}$, the Hilbert space separate-completion of $\mathfrak{M}$ with respect to the scalar product

$$
\langle x \mid y\rangle_{\gamma}=\phi_{\gamma}\left(x^{*} y\right)
$$

by $L_{\gamma}^{2}(\mathfrak{M})$, and denote by $T_{\gamma}: \mathfrak{M} \rightarrow L_{\gamma}^{2}(\mathfrak{M})$ the canonical map.

Claim 1. For any $a, b \in \mathfrak{A}$ and $\gamma \in \Gamma_{\mathfrak{Z}}$ there exists a unique linear continuous $W_{\gamma}^{a b} \in \mathcal{B}\left(L_{\gamma}^{2}(\mathfrak{M})\right)$, such that $W_{\gamma}^{a b} T_{\gamma} x=T_{\gamma}(a x b), \forall x \in \mathfrak{M}$.

Start of by observing that, for any $x \in \mathfrak{M}$, using the inequality $x^{*} a^{*} a x \leq$ $\|a\|^{2} x^{*} x$, and the properties of conditional expectations, we have:

$$
0 \leq E\left((a x b)^{*}(a x b)\right)=E\left(b^{*} x^{*} a^{*} a x b\right) \leq\|a\|^{2} E\left(b^{*} x^{*} x b\right)=\|a\|^{2} b^{*} E\left(x^{*} x\right) b .
$$

Applying the center-valued trace we also have

$$
\left(b^{*} E\left(x^{*} x\right) b\right)^{\natural}=\left(E\left(x^{*} x\right)^{1 / 2} b b^{*} E\left(x^{*} x\right)^{1 / 2}\right)^{\natural} \leq\left(\|b\|^{2} E\left(x^{*} x\right)\right)^{\natural}=\|b\|^{2} E\left(x^{*} x\right)^{\natural},
$$

so going back to (13) we now have

$$
0 \leq E\left((a x b)^{*}(a x b)\right)^{\natural} \leq\|a\|^{2}\|b\|^{2} E\left(x^{*} x\right)^{\natural} .
$$


so if we apply $\gamma$, we now get

$$
0 \leq \phi_{\gamma}\left((a x b)^{*}(a x b)\right) \leq\|a\|^{2}\|b\|^{2} \phi_{\gamma}\left(x^{*} x\right)
$$

Not only that the above inequality proves the implication $T_{\gamma} x=0 \Rightarrow T_{\gamma}(a x b)=0$, which means that one has a correctly defined linear operator

$$
V_{\gamma}^{a b}: \text { Range } T_{\gamma} \ni T_{\gamma} x \longmapsto T_{\gamma}(a x b) \in \text { Range } T_{\gamma},
$$

but it also proves the inequality

$$
\left\|V_{\gamma}^{a b} v\right\|_{L_{\gamma}^{2}(\mathfrak{M})} \leq\|a\| \cdot\|b\| \cdot\|v\|_{L_{\gamma}^{2}(\mathfrak{M})}, \quad \forall v \in \operatorname{Range} T_{\gamma}
$$

so $V_{\gamma}^{a b}$ indeed extends to a linear continuous operator $W_{\gamma}^{a b}$ on $L_{\gamma}^{2}(\mathfrak{M})$. The uniqueness is pretty obvious.

Claim 2. Use the notations as above. For any $\gamma \in \Gamma_{\mathfrak{Z}}$ one has the following implications:

(i) If $a, b \in \mathfrak{A}$ are self-adjoint, then so is $W_{\gamma}^{a b}$.

(ii) If $a, b \in \mathfrak{A}$ are positive, then so is $W_{\gamma}^{a b}$.

To prove (i), we use the scalar product on $L_{\gamma}^{2}(\mathfrak{M})$, which comes from (12), so we must show the identity

$$
\langle x \mid a y b\rangle_{\gamma}=\langle a x b \mid y\rangle_{\gamma}, \forall x, y \in \mathfrak{M}
$$

The right-hand side is

$$
\langle a x b \mid y\rangle_{\gamma}=\gamma\left(E\left((a x b)^{*} y\right)^{\natural}\right) \text {. }
$$

Using the properties of conditional expectations and of the center-valued trace we have

$$
E\left((a x b)^{*} y\right)^{\natural}=E\left(b x^{*} a y\right)^{\natural}=\left(b E\left(x^{*} a y\right)\right)^{\natural}=\left(E\left(x^{*} a y\right) b\right)^{\natural}=E\left(x^{*} a y b\right)^{\natural},
$$

so if we apply $\gamma$, then using (15) we now get

$$
\langle a x b \mid y\rangle_{\gamma}=\gamma\left(E\left(x^{*} a y b\right)^{\natural}\right)=\langle x \mid a y b\rangle_{\gamma},
$$


and (14) is proven.

(ii). This is quite trivial, because we can write $a x b=a^{1 / 2}\left(a^{1 / 2} x b^{1 / 2}\right) b^{1 / 2}$, so if we consider the self-adjoint operator $X=W_{\gamma}^{a^{1 / 2}, b^{1 / 2}} \in \mathcal{B}\left(L_{\gamma}^{2}(\mathfrak{M})\right)$, then clearly $W_{\gamma}^{a b}=X^{2}$, so $W_{\gamma}^{a b}$ is indeed positive.

Having proven Claim 2, let us define, for every $\gamma \in \Gamma_{\mathfrak{Z}}$ the operator $\Theta_{\gamma}=$ $\sum_{j=1}^{n} W_{\gamma}^{a_{j}, b_{j}} \in \mathcal{B}\left(L_{\gamma}^{2}(\mathfrak{M})\right)$, so that

(A) $\Theta_{\gamma} T_{\gamma}=T_{\gamma} \Theta$,

(в) $\Theta_{\gamma}$ is positive.

Suppose now $\lambda \in \mathbb{C} \backslash[0, \infty)$, and $x \in \mathfrak{M}$ is such that $\Theta x=\lambda x$, and let us prove that $x=0$. Using (A) it follows that

$$
\Theta_{\gamma}\left(T_{\gamma} x\right)=T_{\gamma}(\lambda x)=\lambda\left(T_{\gamma} x\right), \quad \forall \gamma \in \Gamma_{\mathfrak{Z}}
$$

By condition (B) this forces $T_{\gamma} x=0$, in $L_{\gamma}^{2}(\mathfrak{M})$, that is, $\phi_{\gamma}\left(x^{*} x\right)=0$, which by definition means

$$
\gamma\left(E\left(x^{*} x\right)^{\natural}\right)=0 \forall \gamma \in \Gamma_{\mathfrak{Z}} .
$$

The above condition forces, of course

$$
E\left(x^{*} x\right)^{\natural}=0(\text { in } \mathfrak{Z}),
$$

which by the faithfulness of both $E$ and the central-valued trace forces $x^{*} x=0$, i.e. $x=0$.

Corollary 3. Assume $\mathfrak{A}$ and $\mathfrak{M}$ are as in Theorem 2 , and $a_{1}, \ldots, a_{n}, b_{1}, \ldots, b_{n} \in \mathfrak{A}$ are positive elements, such that $\sum_{j=1}^{n} a_{j} b_{j}=1$. For any $\lambda \in \mathbb{C}$ and any $\mu \in$ $\mathbb{C} \backslash[0, \infty)$, the equation

$$
\sum_{j=1}^{n} a_{j} z b_{j}=\mu z+\lambda(1-\mu) 1, \quad z \in \mathfrak{M}
$$

has only one solution: $z=\lambda 1$. 
Proof. By Theorem 2, the linear continuous map

$$
\Theta: \mathfrak{M} \ni x \longmapsto \sum_{j=1}^{n} a_{j} x b_{j} \in \mathfrak{A}
$$

has no eigenvalues in $\mathbb{C} \backslash[0, \infty)$. In particular, for any $\mu \in \mathbb{C} \backslash[0, \infty)$ the linear continuous map $\Theta-\mu \mathrm{Id}: \mathfrak{M} \rightarrow \mathfrak{M}$ is one-to-one, thus the only solution of the equation

$$
\Theta(x)=\mu x, \quad x \in \mathfrak{M},
$$

is the trivial one: $x=0$. The rest of the proof is the same as in Corollary 1 .

Corollary 4. If $\mathcal{H}$ is a finite dimensional Hilbert space, and $\mathcal{A}=\left(A_{1}, \ldots, A_{n}\right) \in$ $\mathcal{E}(\mathcal{H})$ is a discrete finite quantum measurement, then the equation

$$
\Lambda_{\mathcal{A}}(B)=I-B, \quad B \in \mathcal{B}(\mathcal{H})
$$

has exactly one solution: $B=\frac{1}{2} I$.

Proof. Immediate from Corollary 3, applied to the (finite dimensional) von Neumann algebras $\mathfrak{A}=\mathfrak{M}=\mathcal{B}(\mathcal{H})$ with $a_{j}=b_{j}=A_{j}^{1 / 2}, \lambda=\frac{1}{2}$ and $\mu=-1$.

Remark. Corollary 4 can also be proven directly using Theorem 3.5 from [1]. Indeed, as noted in the introduction, if $\Lambda_{\mathcal{A}}(B)=I-B$, then $\Lambda_{\mathcal{A}} \circ \Lambda_{\mathcal{A}}(B)=B$. Although the composition $\Phi=\Lambda_{\mathcal{A}} \circ \Lambda_{\mathcal{A}}$ is not a Lüders operation, it is nevertheless a trace-preserving quantum operation, as defined in [1], i.e. of the form

$$
\Phi(B)=\sum_{i, j=1}^{n} E_{i j} B E_{i j}^{*}
$$

with $\sum_{i, j=1}^{n} E_{i j} E_{i j}^{*}=\sum_{i, j=1}^{n} E_{i j}^{*} E_{i j}=I$, where $E_{i j}=A_{i}^{1 / 2} A_{j}^{1 / 2}$. Using Theorem 3.5 from [1], one knows that the condition $\Phi(B)=B$ forces the fact that $B$ commutes with all $E_{i j}$ and $E_{i j}^{*}$. In particular, $B$ commutes with $\sum_{j=1}^{n} E_{i j} E_{i j}^{*}=A_{i}$, so we also get $\Lambda_{\mathcal{A}}(B)=B$. 
Up to this point we were concerned with the eigenvalues of a generalized Lüders operation

$$
\Theta: \mathfrak{M} \ni X \longmapsto \sum_{k=1}^{n} A_{k} X B_{k} \in \mathfrak{M}
$$

within either a unital Banach algebra (as in Theorem 1), or in a special von Neumann algebra setting (as in Theorem 2). It is also natural to consider a slightly weaker version, by restricting $\Theta$ to a two-sided ideal $\mathfrak{J} \subset \mathfrak{M}$, and analyzing the eigenvalues of $\left.\Theta\right|_{\mathfrak{J}}$. In particular, for $\mathfrak{M}=\mathcal{B}(\mathcal{H})$, we may want to consider the pre-dual operation $\left.\Theta\right|_{\mathcal{C}_{1}(\mathcal{H})}$, or $\left.\Theta\right|_{\mathcal{C}_{2}(\mathcal{H})}$, where $\mathcal{C}_{2}(\mathcal{H})$ denotes the Hilbert-Schmidt class. Both of these are special cases of the following easy result.

Corollary 5 (of the proof of Theorem 2). Assume $\mathfrak{M}$ is a von-Neumann algebra with a faithful semi-finite trace $\tau: \mathfrak{M}^{+} \rightarrow[0, \infty]$. Consider the two-sided ideal

$$
\mathfrak{J}=\left\{x \in \mathfrak{M}: \tau\left(x^{*} x\right)<\infty\right\}
$$

If $a_{1}, \ldots, a_{n}, b_{1}, \ldots, b_{n} \in \mathfrak{A}$ are positive elements, then all the eigenvalues of the linear operator

$$
\left.\Theta\right|_{\mathcal{J}}: \mathfrak{J} \ni x \longmapsto \sum_{j=1}^{n} a_{j} x b_{j} \in \mathfrak{J}
$$

are non-negative real numbers.

Proof. The ideal $\mathfrak{J}$ is a pre-Hilbert space, with a unique inner product that satisfies

$$
\langle x \mid x\rangle=\tau\left(x^{*} x\right), \quad x \in \mathfrak{J}
$$

Equivalently, the map $\|\cdot\|_{2}: \mathfrak{J} \rightarrow[0, \infty)$, given by $\|x\|_{2}=\tau\left(x^{*} x\right)^{1 / 2}$, defines a norm, satisfying the Parallelogram Law. As in the proof of Theorem 2, for any two self-adjoint $a, b \in \mathfrak{M}$, the operator

$$
V^{a b}: \mathfrak{J} \ni x \longmapsto a x b \in \mathfrak{J}
$$

is continuous in the $\|\cdot\|_{2}$ norm, and symmetric, i.e.

$$
\left\langle V^{a b} x \mid x\right\rangle=\left\langle x \mid V^{a b} x\right\rangle
$$


therefore it admits a self-adjoint extension $W^{a b} \in \mathcal{B}(\mathcal{K})$, where $\mathcal{K}$ denotes the completion of $\mathcal{J}$. Exactly as in the proof of Theorem 2, if $a, b \in \mathfrak{M}$ are positive, so will be $W^{a b}$, so now the operator $\tilde{\Theta}=\sum_{k=1}^{n} W^{a_{k}, b_{k}} \in \mathcal{B}(\mathcal{K})$ is positive. Of course, by faithfulness, if $\lambda$ is an eigenvalue for $\left.\Theta\right|_{\mathfrak{J}}$, then it is also an eigenvalue for $\tilde{\Theta}$, thus forcing $\lambda \in[0, \infty)$.

Corollary 6. Suppose $p \in[1,2]$ and let $\mathcal{C}_{p}(\mathcal{H})$ denote the associated Schatten-von Neumann class. If $A_{1}, \ldots, A_{n}, B_{1}, \ldots, B_{n} \in \mathcal{B}(\mathcal{H})$ are positive operators, then all eigenvalues of the linear continuous map

$$
\Theta: \mathcal{C}_{p}(\mathcal{H}) \ni X \longmapsto \sum_{j=1}^{n} A_{j} X B_{j} \in \mathcal{C}_{p}(\mathcal{H})
$$

are non-negative real numbers.

Proof. Since $\mathcal{C}_{p}(\mathcal{H}) \subset \mathcal{C}_{2}(\mathcal{H})$, we may assume $p=2$. Then everything follows from the above result applied to $\mathfrak{M}=\mathcal{B}(\mathcal{H})$ and $\tau=$ Trace.

Comments. A. The above results use positivity in an essential way. For arbitrary quantum operations, in the sense of [1], one cannot prevent the appearance of eigenvalues in $\mathbb{C} \backslash[0, \infty)$. For example, if one considers the simplest possible quantum operation

$$
\Phi(X)=U X U^{*}
$$

with $U \in \mathcal{B}(\mathcal{H})$ unitary, then the equation $\Phi(X)=-X$ could have many non-trivial solutions. For instance $U=\left[\begin{array}{cc}1 & 0 \\ 0 & -1\end{array}\right]$ and $X=\left[\begin{array}{cc}0 & 1 \\ 1 & 0\end{array}\right]$.

B. One very important question remains to be considered, in order to obtain a complete understanding of the spectrum of (generalized) Lüders operations: if $\mathcal{A}$ is a discrete finite measurement, is the spectrum of $\Lambda_{\mathcal{A}}: \mathcal{C}_{1}(\mathcal{H}) \rightarrow \mathcal{C}_{1}(\mathcal{H})$ contained in $[0, \infty)$ ?. If this were true, by duality the same would be true for $\mathcal{B}(\mathcal{H})$ in place of $\mathcal{C}_{1}(\mathcal{H})$. 


\section{REFERENCES}

[1] A. Arias, A. Gheondea and S. Gudder, Fixed points of quantum operations, J. Math. Phys. 43(2002) 5872-5881.

[2] P. Busch and J. Singh, Lüders theorem for unsharp quantum effects, Phys. Lett. A, 249 (1998), $10-24$.

[3] S. Gudder, Sequential products of quantum measurements, Preprint 2007.

[4] S. Gudder and G. Nagy, Sequentially independent effects, Proc. A.M.S. 130 (2002), no. 4, $1125-1130$

[5] S. Gudder and G. Nagy, Sequential quantum measurements, J. Math. Phys. 42 (2001), 52125222 .

[6] M. Takesaki, Theory of Operator Algebras I, Springer Verlag, 1979

[7] J. Tomiyama, On the projection of norm one in $W^{*}$-algebras, Proc. Japan Acad. 33 (1957), 608-612.

[8] Y.-Q. Wang, H.-K. Du, N. Zuo A note on sequential products of quantum effects, Preprint 2007

Department of Mathematics, Kansas State university, Manhattan KS 66506, U.S.A.

E-mail address: nagy@math.ksu.edu 